\title{
Plant-plant communication and community of herbivores on tall goldenrod
}

\author{
Kaori Shiojiri ${ }^{1}$, Satomi Ishizaki ${ }^{2}$, and Yoshino Ando ${ }^{3}$ \\ ${ }^{1}$ Ryukoku University \\ ${ }^{2}$ Niigata University Faculty of Agriculture \\ ${ }^{3}$ Hokkaido University Field Science Center for Northern Biosphere
}

November 13, 2020

\begin{abstract}
1. The volatiles from damaged plants induce defense in neighboring plants. The phenomenon is called plant-plant communication, plant talk or plant eavesdropping. Plant-plant communication has been reported to be stronger between kin plants than genetically far plants in sagebrush. 2. Why do plants distinguish volatiles from kin or genetically far plants? We hypothesize that plants respond only to important conditions; the induced defense is not free of cost for the plant. To clarify the hypothesis, we conducted experiments and investigations using goldenrod of 4 different genotypes. 3. The arthropods community on tall goldenrods were different among 4 genotypes. The response to volatiles was stronger from genetically close plants to the emitter than from genetically distant plants from the emitter. The volatiles from each genotype of goldenrods were different; and they were categorized accordingly. Moreover, the arthropod community on each genotype of goldenrods were different. 4. Synthesis: Our results support the hypothesis: goldenrods respond to volatiles from genetically close plants because they would have similar arthropod species. These results are important clues elucidating adaptive significance of plant-plant communication.
\end{abstract}

\section{Hosted file}

shiojiri et al. (text).pdf available at https://authorea.com/users/375542/articles/492734plant-plant-communication-and-community-of-herbivores-on-tall-goldenrod 